\title{
Targeting JNK for therapeutic depletion of stem-like glioblastoma cells
}

SUBJECT AREAS:

CANCER

CANCER STEM CELLS

MEDICAL RESEARCH

CELL BIOLOGY

Received

19 April 2012

Accepted

27 June 2012

Published

19 July 2012

Correspondence and requests for materials should be addressed to C.K. (ckitanak@med. id.yamagata-u.ac.jp) or A.S. (asato@med. id.yamagata-u.ac.jp)

* These authors contributed equally to this work.
Ken-ichiro Matsuda ${ }^{1,2 *}$, Atsushi Sato ${ }^{1,2 *}$, Masashi Okada', Keita Shibuya', Shizuka Seino', Kaori Suzuki ${ }^{13}$, Eriko Watanabe', Yoshitaka Narita ${ }^{3}$, Soichiro Shibui' ${ }^{3}$, Takamasa Kayama ${ }^{2}$ \& Chifumi Kitanaka ${ }^{1,4,5}$

'Department of Molecular Cancer Science, ${ }^{2}$ Department of Neurosurgery, Yamagata University School of Medicine, Yamagata, 990-9585, Japan, ${ }^{3}$ Neurosurgery Division, National Cancer Center Hospital, Tokyo, 104-0045, Japan, ${ }^{4}$ Oncology Research Center, Research Institute for Advanced Molecular Epidemiology, Yamagata University, Yamagata, 990-9585, Japan, ${ }^{5} \mathrm{Global}$ COE program for Medical Sciences, Japan Society for Promotion of Science, Tokyo, 102-8472, Japan.

Control of the stem-like tumour cell population is considered key to realizing the long-term survival of patients with glioblastoma, one of the most devastating human malignancies. To date, possible therapeutic targets and targeting methods have been described, but none has yet proven to target stem-like glioblastoma cells in the brain to the extent necessary to provide a survival benefit. Here we show that targeting JNK in vivo, the activity of which is required for the maintenance of stem-like glioblastoma cells, via transient, systemic administration of a small-molecule JNK inhibitor depletes the self-renewing and tumour-initiating populations within established tumours, inhibits tumour formation by stem-like glioblastoma cells in the brain, and provide substantial survival benefit without evidence of adverse events. Our findings not only implicate JNK in the maintenance of stem-like glioblastoma cells but also demonstrate that JNK is a viable, clinically relevant therapeutic target in the control of stem-like glioblastoma cells.

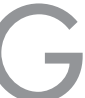

lioblastoma multiforme, the most common primary brain neoplasm in adults, is among the deadliest of all human cancers $^{1-5}$. Advancement in the treatment of glioblastoma has lagged far behind that of other cancer types and stagnated over decades, except for the small but significant progress recently made by the introduction of temozolomide, a new alkylating chemotherapeutic agent ${ }^{2-6}$. The current standard of treatment for glioblastoma consists of maximal surgical resection followed by radiotherapy with concomitant and adjuvant temozolomide ${ }^{1,6,7}$. However, despite optimal treatment, even in cases in which the tumour responds well to initial treatment and appears to have disappeared on follow-up scanning, recurrence is inevitable and fatal, with only few patients surviving beyond 5 years ${ }^{1,3,4,7,8}$. Such a dismal clinical picture of glioblastoma points to the possibility that a small but significant proportion of tumour cells with high tumour-initiating potential retain the capacity to tactfully evade all forms of radical treatment. Adding further complexity to the treatment of glioblastoma are its highly invasive nature and the presence of the blood-brain barrier, which limits the access of chemical substances into the brain parenchyma ${ }^{4,6,9-12}$. After leaving the bulk tumour where the blood-brain barrier is disrupted, glioblastoma cells disperse into unresectable brain regions far beyond the margin of the radiation field, where they are securely protected from chemicals by the intact blood-brain barrier ${ }^{4,69-12}$. Thus, to control glioblastoma and realize long-term survival and, ultimately, cure of patients suffering from this devastating disease, it is essential to develop novel measures to selectively kill such therapy-resistant populations of glioblastoma cells or deprive them of their tumour-initiating potential despite this natural barrier.

The cancer stem cell hypothesis holds that tumours are heterogeneous, being composed of both a rare subpopulation of cancer stem cells with the ability to self-renew indefinitely and initiate tumour formation and a majority population of tumour cells with limited ability to divide, and therefore incapable of initiating tumour formation $^{2,3,5,13,14}$. Although recent findings indicate that this hypothesis may not apply to all cancer types ${ }^{14}$, accumulating evidence indicates that it does apply to glioblastomas, as they appear to contain a cancer stem cell population ${ }^{2-5}$. Of importance, these hypothetical cancer stem cells possess both stem-like properties (e.g., self-renewal capacity and multipotency of differentiation) and tumour-initiating potential ${ }^{14,15}$. Although it remains unknown why such seemingly disparate characteristics should co-localize within the same cells, a wealth of experimental evidence indicates that they indeed do $\mathrm{so}^{2,5}$, suggesting that the characteristics of stem-like properties and tumour-initiating potential are very closely linked. Thus, both the hypothesis and evidence support the idea that molecules involved in the regulation of these stem-like properties are attractive targets in controlling the tumour-initiating potential of cancer 
(stem) cells. Another key tenet of the hypothesis is that differentiation of cancer stem cell into non-stem cancer cell is a one-way, irreversible process $^{13,14}$. Although this tenet has not yet been fully proven experimentally ${ }^{14}$, it implies that after the successful differentiation of cancer stem cells into non-stem cancer cells within a tumour, the tumour would permanently lose the ability to form recurrent tumours even without further, continuous treatment.

Encouraged by such a groundbreaking possibility, we undertook this study to search for molecules involved in the regulation of the stem-like properties of glioblastoma cells, with the clear intention to identify druggable molecular targets together with drugs targeting the molecules. By depriving, ideally irreversibly, glioblastoma cells of their tumour-initiating potential, such drugs would greatly contribute to the long-term survival of glioblastoma patients by preventing fatal recurrence.

\section{Results}

Differential activation of the JNK pathway in self-renewing and differentiated stem-like glioblastoma cells. To identify candidate regulators of the stem-like properties (i.e., the capacity to self-renew and to undergo multi-lineage differentiation, see Supplementary Fig. 1 for characterization of the stem-like glioblastoma cells used in this study) of stem-like glioblastoma cells, we searched for molecules differentially expressed and/or activated in self-renewing and differentiated stem-like glioblastoma cells. We found that, compared to their differentiated counterparts, self-renewing stem-like glioblastoma cells have elevated levels of JNK phosphorylation at the activating phosphorylation sites. We also found that the increased JNK phosphorylation is accompanied by increased c-Jun phosphorylation at the cognate JNK phosphorylation site, indicating increased JNK pathway activation in self-renewing cells (Fig. 1a). Notably, whereas the differential activation status of other signalling pathways implicated in glioblastoma biology and of related MAPK superfamily members ${ }^{11,16}$ was inconsistent and varied depending on the cell line tested (Fig. 1b), the JNK pathway was consistently activated in self-renewing cells relative to differentiated cells in all the stem-like glioblastoma cell lines tested including those directly derived from glioblastoma patients (patient-derived stem-like glioblastoma cells) as well as those established from conventional, serum-cultured cell lines (Fig. 1a).

a

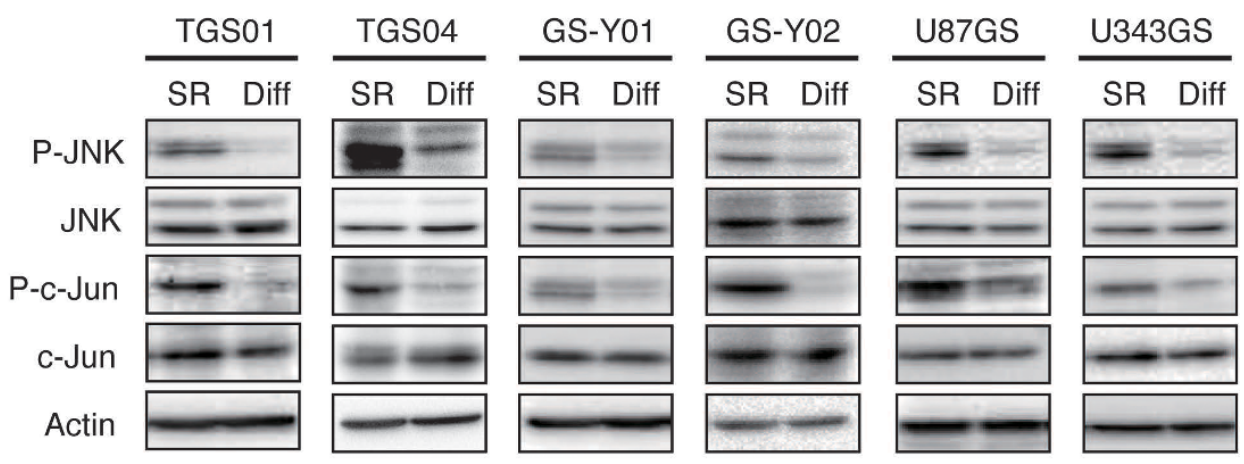

b

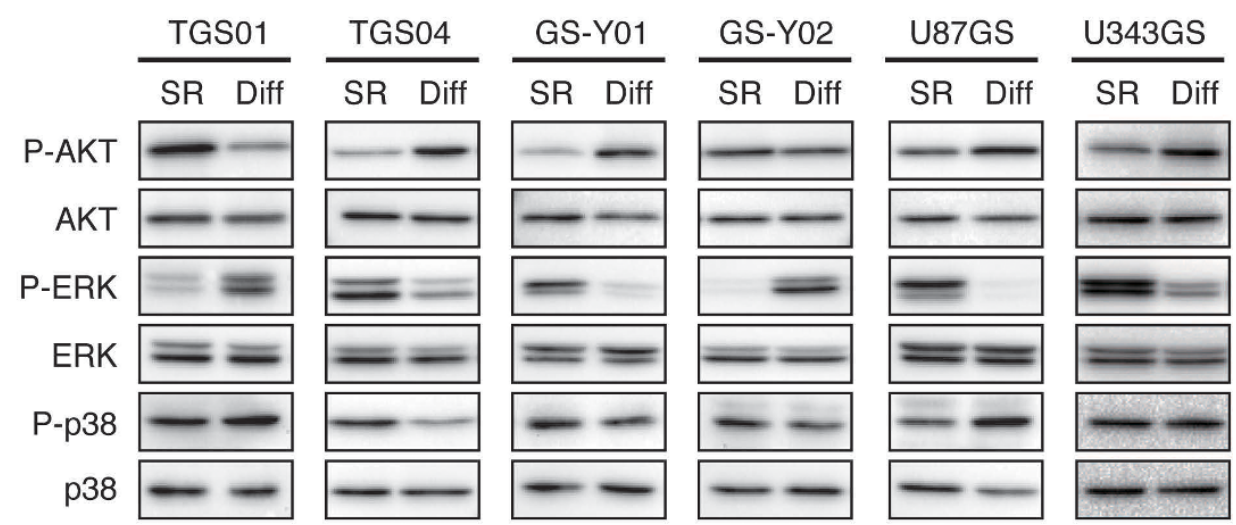

Figure $1 \mid$ Differential activation of the JNK signalling pathway in self-renewing and differentiated stem-like glioblastoma cells. (a-b) Self-renewing (maintained under the monolayer stem cell culture condition; SR) and differentiated (cultured under the differentiation-inducing condition for 1 week; Diff) stem-like glioblastoma cells were subjected to immunoblot analysis to examine the activation/phosphorylation status of the JNK signalling pathway (a) and that of AKT, ERK, and p38 (b). 
JNK is required for self-renewal and prevention of stem-like glioblastoma cell differentiation. Prompted by observation of a uniform JNK pathway activation in self-renewing stem-like glioblastoma cells, we next investigated whether JNK is involved in the maintenance of the stem-like properties of self-renewing cells. We first tested the effect of SP600125, a "reversible", ATP-competitive inhibitor of $\mathrm{JNK}^{17}$, on the ability of stem-like glioblastoma cells to self-renew themselves as tumourspheres at concentrations that inhibited c-Jun phosphorylation (and expression) (Fig. 2b) but not cellular viability (Supplementary Fig. 2). Whereas the cells pretreated with the control vehicle maintained the ability to form tumourspheres over serial passages, stem-like glioblastoma cells pretreated with SP600125 showed reduced ability to form tumourspheres even in the absence of the inhibitor (Fig. 2a), suggesting that transient JNK inhibition had deprived the cells of their self-renewing capacity. To determine whether such decreased tumoursphere formation truly reflects loss of stem-like properties, the expression of stem cell and differentiation markers was next examined. SP600125 treatment was found to cause decreased expression of stem cell markers such as Nestin, Sox2, and Musashi-1, accompanied by increased expression of the differentiation markers, glial fibrillary acidic protein (GFAP) and BIII-tubulin (Fig. 2c). These changes in marker expression level reflected the change in the ratio of undifferentiated to differentiated cell populations, as revealed by immunocytochemical analysis (Fig. 2d). The results of these pharmacological inhibitor assays were confirmed by subsequent knockdown experiments. Unexpectedly, we found that knockdown of either JNK1 or JNK2 in stem-like glioblastoma cells is sufficient to effectively inhibit the JNK pathway activity (Fig. 2f). This finding may be in line with a previous study using mouse embryonic fibroblasts null for either JNK1 or JNK2, which found that both JNK1 and JNK2 are required for JNK pathway activation ${ }^{18}$. We therefore knocked down either JNK1 or JNK2 singly in the following experiments. The results indicate that, similar to those regarding SP600125, short-interfering RNA (siRNA)-mediated knockdown of JNK1 or JNK2 inhibits tumoursphere formation (Fig. 2e) and stem cell marker expression while inducing the expression of differentiation markers (Fig. 2f). Intriguingly, we found that expression of the FOXO1 transcription factor but not of FOXO3, which has previously been implicated in the differentiation of stem-like glioblastoma cells ${ }^{19}$, is upregulated accompanied by its nuclear translocation upon JNK inhibition in stem-like glioblastoma cells (Supplementary Figs. 3 and 4). We also found that FOXO1 knockdown inhibits commitment of stemlike glioblastoma cells to differentiation (Supplementary Fig. 3). These results suggest that prevention of FOXO1 activation is at least in part responsible for the JNK-mediated maintenance of stem-like glioblastoma cells. Collectively, the data suggest that continuous, uninterrupted activation of the JNK pathway is essential for preventing premature activation of the differentiation-inducing program, and therefore, for the maintenance of the self-renewal capacity of glioblastoma cells. Strikingly, such JNK dependence was confirmed in all 10 patient-derived stem-like glioblastoma cell lines tested in this study, which had been originally established in 3 independent institutions, as well as in the 2 stem-like cell lines established from conventional, serum-cultured glioblastoma cell lines (Supplementary Figs. 5-7). Furthermore, JNK was found to be required for tumoursphere formation and/or maintenance of the undifferentiated state in putative stem-like glioblastoma cells that ultimately failed to become established cell lines (Supplementary Fig. 8), in support of the idea that JNK dependence of self-renewal is not a unique feature of established cell lines. Thus, the critical role of JNK in the control of self-renewal and differentiation could be a cardinal feature shared by stem-like glioblastoma cells.

JNK inhibition in vitro deprives stem-like glioblastoma cells of tumour-initiating potential. Although the existence of a hierarchical, irreversible relationship between stem-like and differentiated cancer cells within a tumour remains to be conclusively demonstrated, a large body of evidence accumulated over many years indicates that, at least in certain types of human cancers, there exists cellular heterogeneity within tumours and that the stem-like, immature phenotype is more closely coupled with the characteristic of high tumour-initiating potential compared to the differentiated phenotype ${ }^{14}$. Indeed, accumulating evidence indicates that it is actually the case with glioblastoma ${ }^{2,5}$. We therefore asked next if such association between the stem-like phenotype and the characteristic of tumour-initiating potential applies to stem-like glioblastoma cells before and after artificial induction of differentiation by JNK inhibition. To this end, we first implanted patient-derived stem-like cells (TGS01) pretreated with or without SP600125 subcutaneously into immunocompromised mice so that we can monitor the kinetics of tumour growth over time (Fig. 3a). Tumour formation by TGS01 cells pretreated with SP600125 in vitro was markedly delayed compared to that of cells pretreated with the control vehicle. Direct measurement of subcutaneous tumour weight also indicated inhibited tumour growth of the SP600125-treated cells. Similar inhibition of tumour growth was observed when TGS01 cells were implanted after transient knockdown of either JNK1 or JNK2 (Fig. 3b), demonstrating that JNK is required for the maintenance of tumour-initiating potential just as it is required for the maintenance of stem-like properties. The results of similar experiments conducted using stem-like cells (U87GS) derived from the U87 glioblastoma cell line were essentially similar (Supplementary Fig. 9a, b), suggesting that JNK dependence of the tumour-initiating potential of stem-like cells may be a robust mechanism that can be maintained over long-term serum culturing. Of note, when the bulk, serum-cultured U87 cells were subjected to the xenograft assay, the identical SP600125 pretreatment protocol, which substantially delayed and even prevented tumour formation by stem-like U87GS cells, had only modest slowing effect on the tumour growth of serum-cultured U87 cells (Supplementary Fig. 10). Thus, JNK likely plays a much more significant role in the maintenance of tumour-initiating potential in stem-like cells compared to non-stem glioblastoma cells. We next confirmed the JNK dependence of the tumour-initiating potential of stem-like glioblastoma cells in the orthotopic context. Whereas intracerebral implantation of patient-derived cells (TGS01, $\left.1 \times 10^{4}\right)$ pretreated with the control vehicle resulted in formation of invariably fatal brain tumours (i.e., $100 \%$ mortality of all 5 mice examined), intracerebral implantation of cells pretreated with SP600125 in vitro resulted in the death of only 1 of the 5 mice examined, with the remaining 4 mice surviving longer than 1 year without any neurological symptoms (Fig. 3c). Histological analysis of mouse brains demonstrated formation of large brain tumours in the mice that had received controltreated cells but no tumour formation in the brains of mice that had received SP600125-treated cells (Fig. 3c). Again, essentially similar results were obtained when U87GS cells were used (Supplementary Fig. 9c). Thus, JNK is required for not only maintenance of stem-like properties but also of the tumour-initiating potential of stem-like glioblastoma cells.

Depletion of self-renewing and tumour-initiating glioblastoma cells by JNK inhibition in vivo. Having established the essential role of JNK in the maintenance of the tumour-initiating potential of stem-like glioblastoma cells, we next sought to determine if JNK could be an in vivo target in controlling the tumour-initiating potential of glioblastoma cells. To this end, we tested the effect of systemic administration of SP600125 on tumour formation by stemlike glioblastoma cells. We started in this study from a much less intense, short-term regimen (intraperitoneal administration of SP 600125 at $40 \mathrm{mg} / \mathrm{kg} / \mathrm{day}$ for 5 consecutive days) compared to the regimen used in a previous study (daily intraperitoneal administration of SP600125 at $50 \mathrm{mg} / \mathrm{kg} /$ day for 1 month to nude 
a
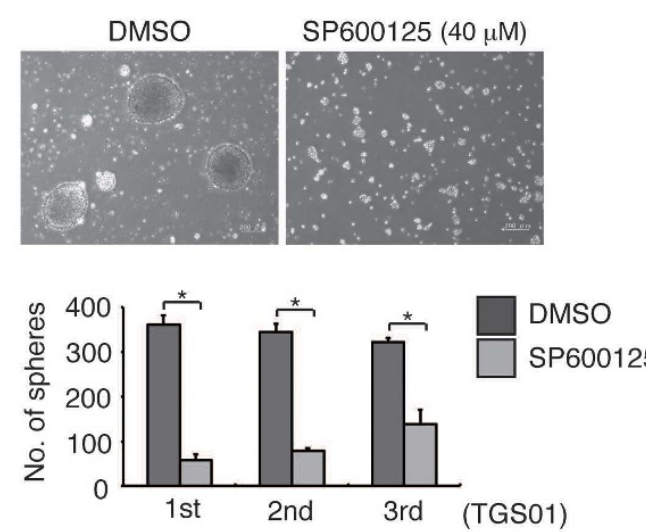

b

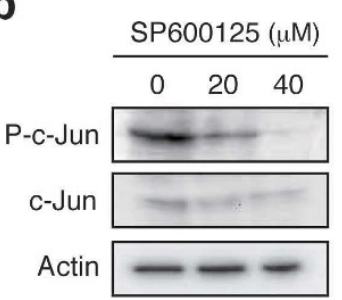

(TGS01)

d

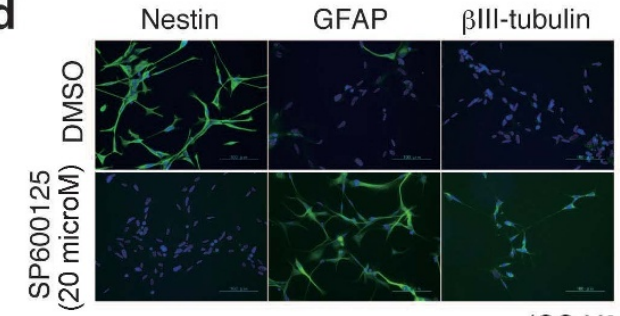

Nestin

GFAP

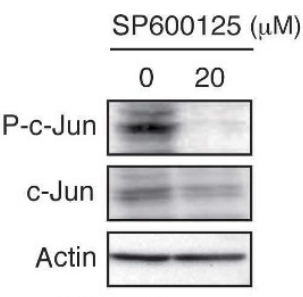

(GS-Y01)

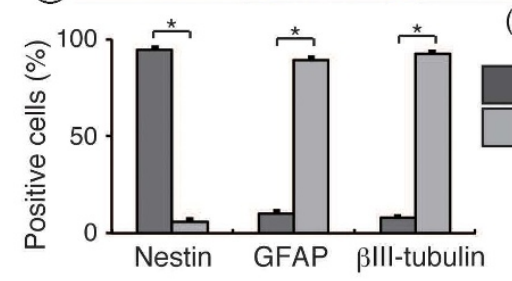

(GS-Y01)

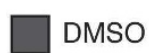

SP600125 (20 microM)

e

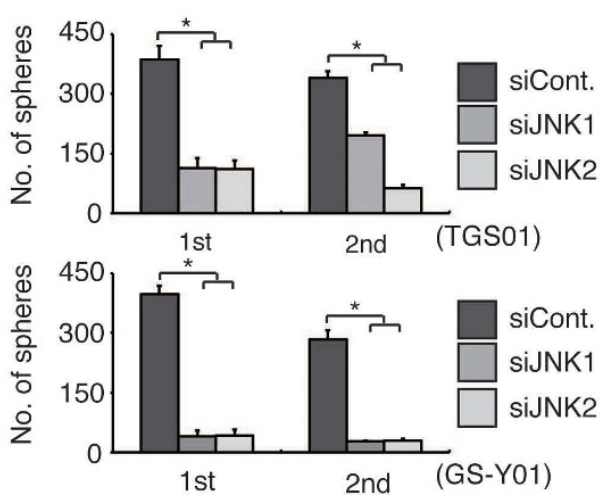

DMSO

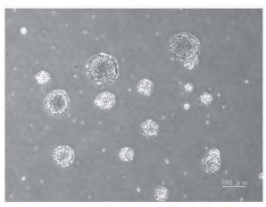

SP600125 (20 $\mu \mathrm{M})$
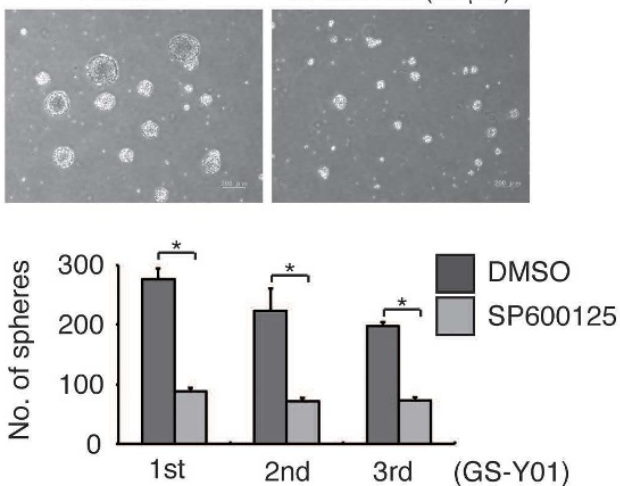

C

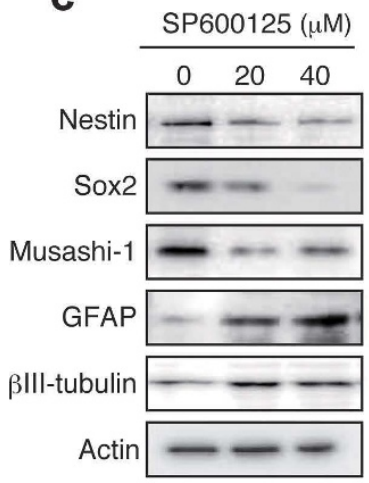

(TGS01)

f

siRNA Cont. JNK1 JNK2

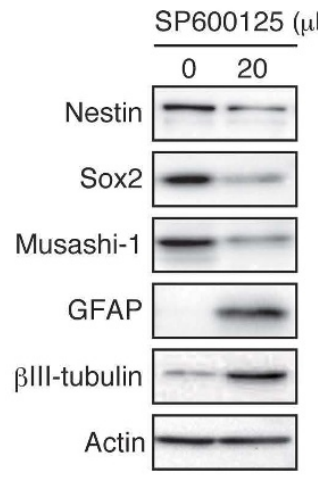

(GS-Y01)

\section{siRNA Cont. JNK1 JNK2}

JNK1

JNK2

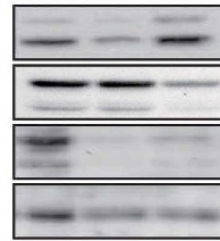

C-Jun

Nestin

Sox2

Musashi-1
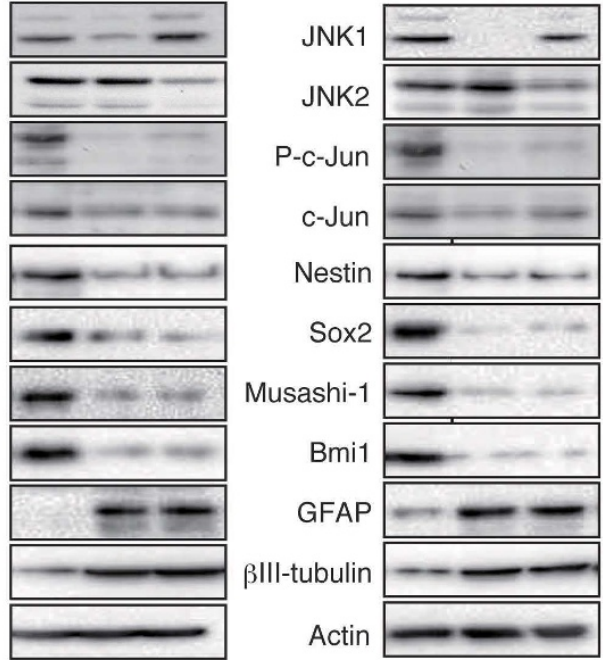

Actin

(TGS01)

(GS-Y01)

Figure $2 \mid$ JNK is required for the maintenance of self-renewal capacity of stem-like glioblastoma cells. (a) Stem-like glioblastoma cells cultured in the presence of SP600125 ( $40 \mu \mathrm{M}, 7$ days for TGS01; $20 \mu \mathrm{M}, 3$ days for GS-Y01) were subjected after washout of the inhibitor to serial (i.e., primary to tertiary) sphere formation assays in the absence of SP600125. Top, phase-contrast micrographs of the primary spheres (scale bars, $200 \mu \mathrm{m})$. Bottom, number of tumourspheres formed (mean \pm s.d. from 3 experiments). ${ }^{*} P<0.05$. (b-d) TGS01 and GS-Y01 cells were cultured in the presence of the indicated concentrations of SP600125 for 7 and 3 days, respectively. Cells were then subjected to immunoblot analysis for the expression of phospho-c-Jun (b) and stem cell and differentiation markers (c) and to immunofluorescence staining (green) of nestin, GFAP, and $\beta$ III-tubulin (d). In (d), nuclei were counterstained with Hoechst 33342 (blue), and the values in the graph represent mean \pm s.d. from 3 experiments. Scale bars, $100 \mu \mathrm{m}$. $* P<0.05$. (e-f) TGS01 and GS-Y01 cells were transiently transfected with siRNAs against JNK1 or JNK2, or with a control siRNA. Cells were then subjected to serial sphere formation assays ( $\mathrm{e}$, values represent mean \pm s.d. from 3 experiments)) or to immunoblot analysis to examine the JNK signalling pathway ( 3 days after transfection) and stem cell/differentiation status ( 7 and 3 days after transfection for TGS01 and GS-Y01, respectively) (f). $* P<0.05$. 
a
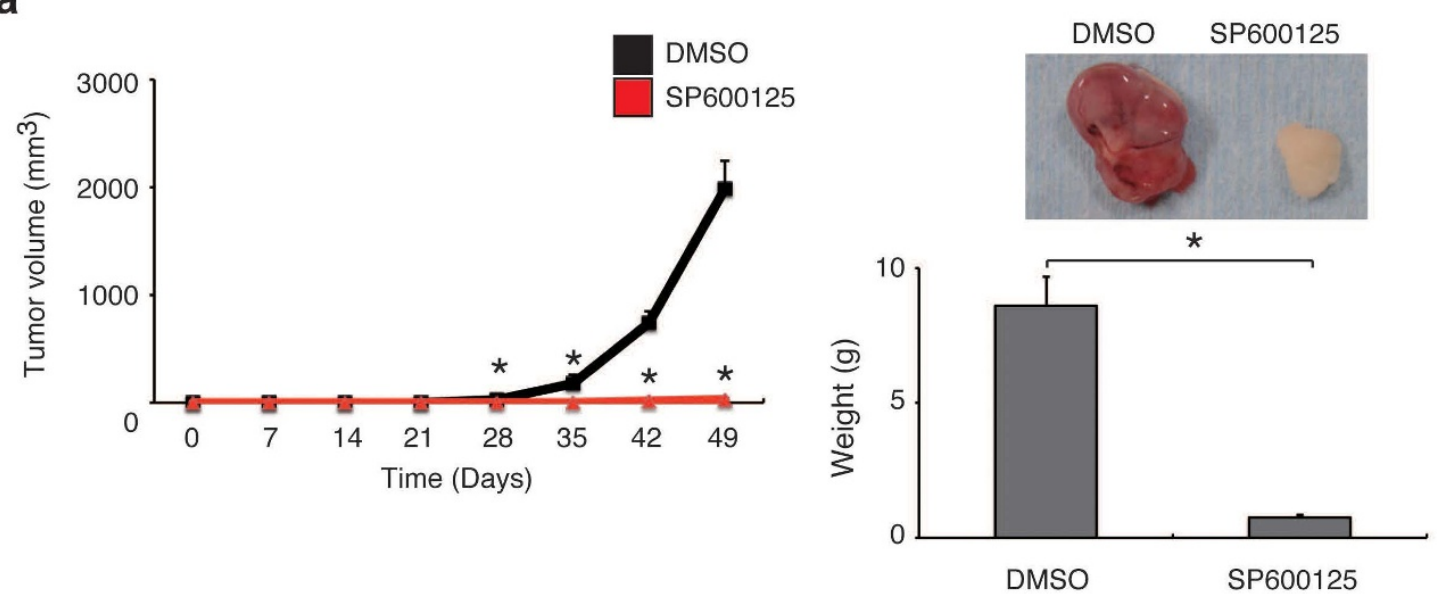

b
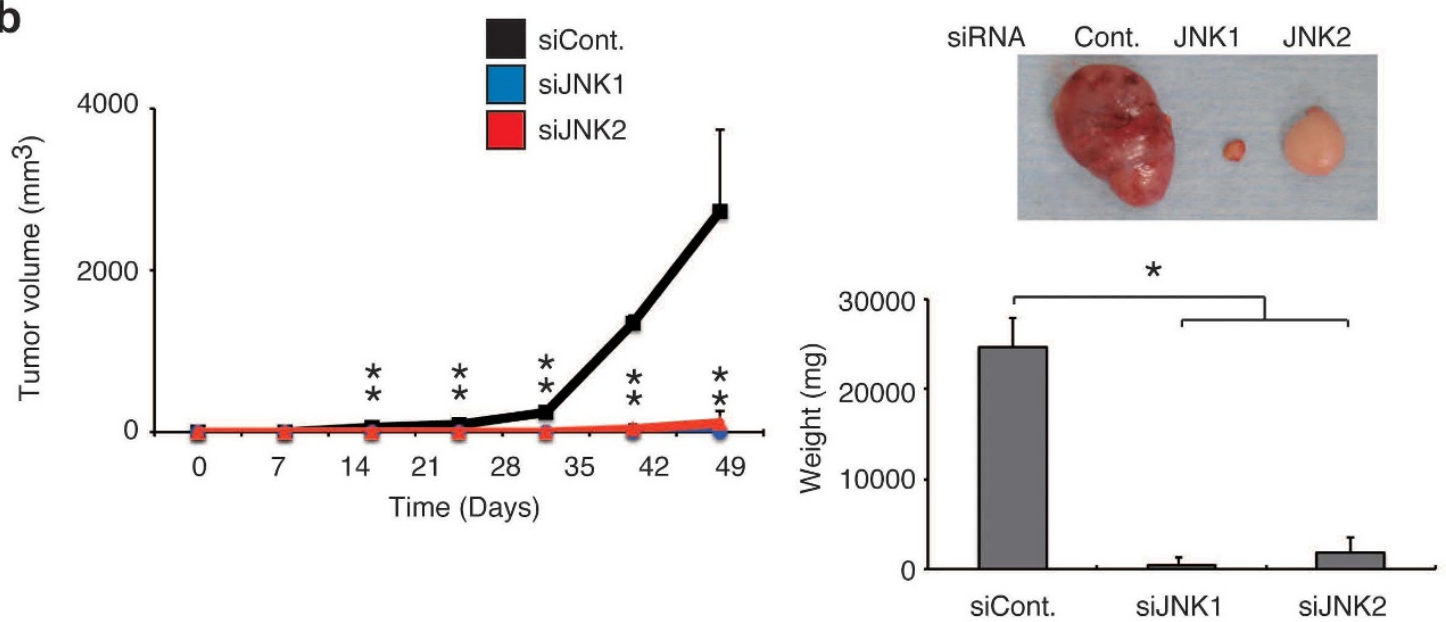

C
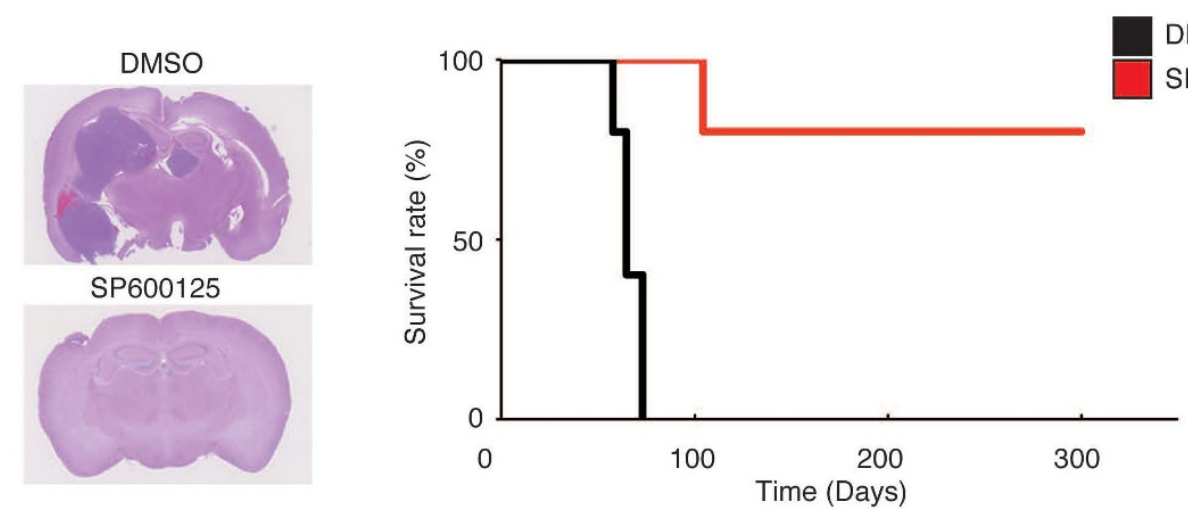

DMSO $\mathrm{SP} 600125]$ *

Figure $3 \mid$ JNK is required for the maintenance of the tumour-initiating potential of stem-like glioblastoma cells. (a) TGS 01 cells $\left(1 \times 10^{6}\right)$ pretreated with SP600125 $(40 \mu \mathrm{M})$ or the control vehicle for 7 days were implanted subcutaneously into the right flank of nude mice (5 mice per group). Tumour volume was measured at the indicated time points and the results presented as mean tumour volume \pm s.d. (left). At the end of the observation period, mice were sacrificed and the weight of subcutaneous tumours was measured (right bottom). Representative subcutaneous tumours formed by SP600125and control-treated stem-like glioblastoma cells are shown (top right). ${ }^{*} P<0.05$. (b) TGS01 cells $\left(1 \times 10^{6}\right)$ transiently transfected with siRNAs against JNK1 or JNK2, or with a control siRNA were implanted subcutaneously into the right flank of nude mice 7 days after transfection ( 5 mice per group). Mice were then analysed as in (a). ${ }^{*} P<0.05$. (c) A Kaplan-Meier plot (right) showing the survival of mice ( 5 mice per group) after intracranial implantation of TGS01 cells $\left(1 \times 10^{4}\right)$ pretreated with SP600125 $(40 \mu \mathrm{M})$ or the control vehicle (DMSO) for 7 days. Representative images of haematoxylin and eosin staining of the brain sections from mice sacrificed at 60 days after implantation are shown $($ left $) .{ }^{*} P<0.05$.

mice $)^{20}$, and evaluated the effectiveness of the regimen against subcutaneous tumour formation to see if intensification of the treatment schedule is needed. Rather unexpectedly, even with this starting, less intense regimen of drug administration, we observed a significant inhibitory effect of SP600125 treatment compared to the control treatment against tumour formation either by stem-like 
glioblastoma cells directly derived from a patient (TGS01; Fig. 4a) or by stem-like U87GS cells derived from the conventional, serumcultured cell line U87 (Supplementary Fig. 9d).

We then wished to ask whether we could control the self-renewing, stem-like cell population within established glioblastoma xenografts with this SP600125 treatment protocol. Mice bearing a subcutaneous glioblastoma xenograft pre-established by implantation of patient-derived stem-like cells (TGS01) were administered daily intraperitoneal injection of SP600125 (40 mg/kg) or the control vehicle for 5 consecutive days after the tumour had become 8-9 $\mathrm{mm}$ in diameter. After 5 days of administration, the subcutaneous tumour was excised, dissociated, and subjected to tumoursphere formation assay to evaluate the number of stem-like cells capable of self-renewing as spheres. Compared to the control-treated tumours, which consistently gave rise to large, actively proliferating tumourspheres with stem-like properties (Supplementary Fig. 11), the SP600125-treated tumours produced few non-adherent tumourspheres, and most of the tumour cells died or remained attached to the culture plate without proliferating (Fig. $4 \mathrm{~b}$ ). Strikingly, when cells derived from tumours treated in vivo either with the control vehicle or SP600125 using the same protocol were seeded and cultured in the presence of serum, they began to grow noticeably and showed similar growth curves irrespective of prior treatment (Supplementary Fig. 12). Thus, the results suggest that the in vivo SP600125 treatment protocol used here selectively depletes the self-renewing, stem-like cell population without having any growth inhibitory effects on bulk tumour cells.

Having found that the in vivo SP600125 treatment protocol depletes the stem-like cell population within glioblastoma xenografts, we next sought to determine if we could eliminate the tumour-initiating population within established tumours using the same treatment protocol. Tumour cells obtained by dissociation of the TGS01 glioblastoma xenografts treated with either SP600125 or the control vehicle in vivo were directly transplanted subcutaneously for secondary tumour formation. Whereas all 3 of the 3 mice transplanted with cells derived from the control-treated tumours developed secondary tumours within 1 week, 2 of the 3 mice transplanted with cells from the SP600125-treated tumours remained tumour free at 3 weeks and 1 mouse remained tumour free at 4 weeks (Fig. 4c). Similar experiments using stem-like U87GS cells revealed that the same treatment protocol completely prevents secondary tumour formation (Supplementary Fig. 9e).

We then went on to confirm the inhibitory effect of in vivo JNK inhibition on secondary tumour formation in the brain. To perform quantitative measurement of the extent of SP600125-mediated depletion of the tumour-initiating population, cells obtained by dissociation of the tumours treated in vivo with either SP600125 or the control vehicle were transplanted, after serial dilution, orthotopically into the brains of immunocompromised mice for secondary tumour formation (Fig. 4d). All mice that had received cells from the controltreated tumours $\left(1 \times 10^{5}-1 \times 10^{3}\right.$ cells) died within 2 months from brain tumour burden, with the survival period found to be inversely correlated with the number of cells transplanted. In stark contrast, brain tumour death of mice that had received cells from the SP600125-treated tumours was delayed or even prevented: mice that had received $1 \times 10^{5}$ of the SP600125-treated tumour cells survived just as long as those that had received $1 \times 10^{4}$ of the control-treated tumour cells, with 1 of the 3 mice that had received $1 \times 10^{4}$ of the SP600125-treated tumour cells and 3 of the 3 mice that had received $1 \times 10^{3}$ of the SP600125-treated tumour cells remaining alive with no sign of brain tumour burden at 10 months after transplantation. These results indicate that JNK inhibition with the in vivo SP600125 treatment protocol (intraperitoneal injection of $40 \mathrm{mg} /$ $\mathrm{kg} /$ day of SP600125 for 5 consecutive days) depletes the tumourinitiating population within established glioblastoma xenografts by one or more orders of magnitude. The results of a similar experiment using temozolomide at a maximally tolerable dose (i.e., sublethal dose) demonstrated that temozolomide has no discernible inhibitory effect on secondary brain tumour formation by TGS01 cells (Supplementary Fig. 13). Although the results alone do not exclude the possibility that temozolomide has the reported ability to target the stem-like, tumour-initiating subpopulation of glioblastoma cells $^{21}$, they clearly indicate that SP600125 treatment is capable of effectively eliminating in vivo the tumour-initiating population that even temozolomide, the first-line chemotherapeutic agent in current glioblastoma treatment, fails to target.

Targeting stem-like glioblastoma cells in the brain by systemic JNK inhibitor administration. The inhibitory effect of systemic administration of SP600125 on the JNK activity in the brain parenchyma has been well documented in the context of treatment models for various neurological conditions ${ }^{22,23}$. In consideration of this fact, we examined, finally, whether SP600125 administered intraperitoneally deprives orthotopically implanted stem-like glioblastoma cells of their tumour-initiating potential to the extent necessary to provide a survival benefit. The results of pilot orthotopic xenograft experiments involving implantation of serially diluted stem-like glioblastoma cells suggested that reduction of the quantity of stem-like cells by one order of magnitude results in only negligible or minimal survival benefit, depending on the cell line and experimental condition (Supplementary Fig. 14). Therefore, we intensified the SP600125 treatment protocol by extending the treatment period (10 consecutive days instead of 5), with the daily dose fixed at $40 \mathrm{mg} / \mathrm{kg} /$ day. When mice that had undergone intracerebral implantation of TGS01 stem-like glioblastoma cells $\left(1 \times 10^{4}\right)$ were treated with either the control vehicle or SP600125 in accordance with this new, 10-day protocol, survival was significantly enhanced by the SP600125 treatment compared to the control treatment (Fig. 5a). Specifically, SP600125 treatment extended the median survival time by 30 days (22 days for control vs. 52 days for $\mathrm{SP} 600125$ ), suggesting that it had reduced the tumourinitiating population by more than 2 orders of magnitude (Fig. $5 \mathrm{a}$; also see Supplementary Fig. 14 for reference). In line with the in vitro data showing that JNK is required for the maintenance of the stemlike properties in all the stem-like glioblastoma cells tested (Fig. 2 and Supplementary Figs. 5-8), significant survival benefits (roughly estimated to reflect a 10 - to 100 -fold reduction in the tumourinitiating population) were also observed in all similar orthotopic xenograft experiments conducted thus far using other patientderived (GS-Y01 and TGS04; Fig. 5b and 5c, respectively) and conventional cell-line derived (U87GS; Fig. 5d) stem-like glioblastoma cells. In a parallel experiment, cohorts of mice not undergoing the implantation procedure were treated with either the control vehicle or SP600125 according to the 10-day protocol to monitor any possible adverse events. All mice survived beyond 12 months after treatment, with no significant differences found in general health status as assessed by body weight and survival (Supplementary Fig. 15) and in cognitive function as assessed by Y-maze test (Supplementary Fig. 16) between the control and SP600125 treatment groups.

\section{Discussion}

In contrast to conventional glioblastoma therapies, which are directed chiefly at reduction of bulk tumours (where the blood-brain barrier is disrupted as shown by take-up of contrast material) and invariably associated with tumour recurrence, future curative therapies should be directed, in addition, at elimination of the tumourinitiating glioblastoma cells that infiltrate deep into unresectable brain regions protected by the intact blood-brain barrier. Thus, a curative anti-glioblastoma therapeutic agent must have the ability to be distributed throughout the brain parenchyma at a concentration sufficient to kill or deprive them of their tumour-initiating 
a

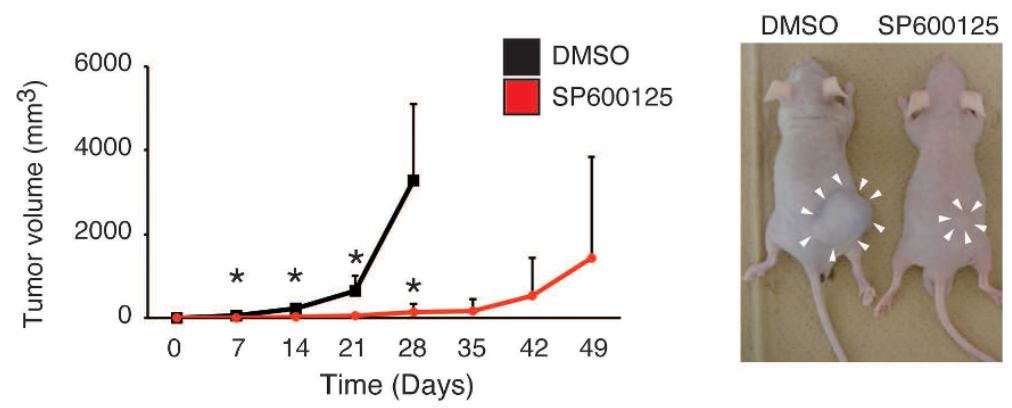

b
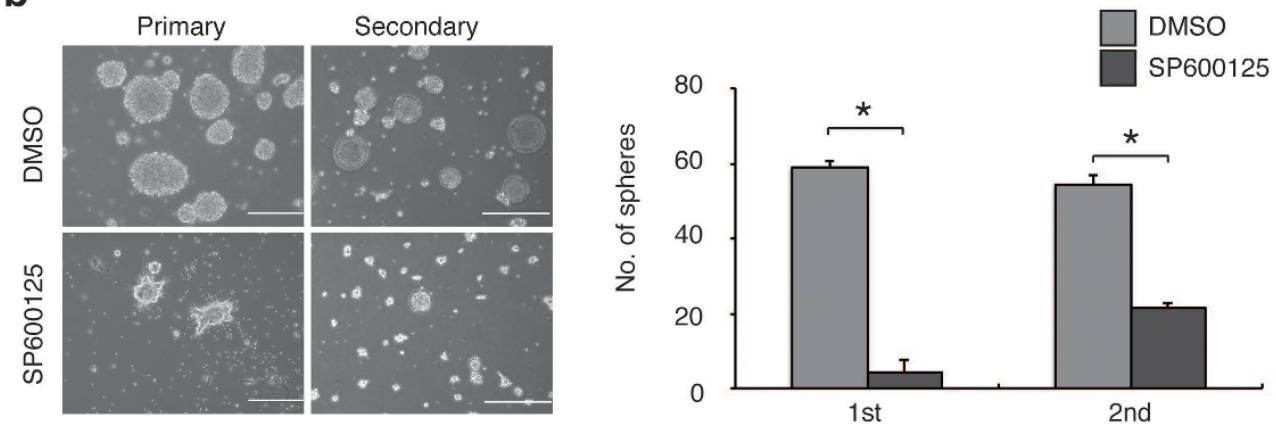

C
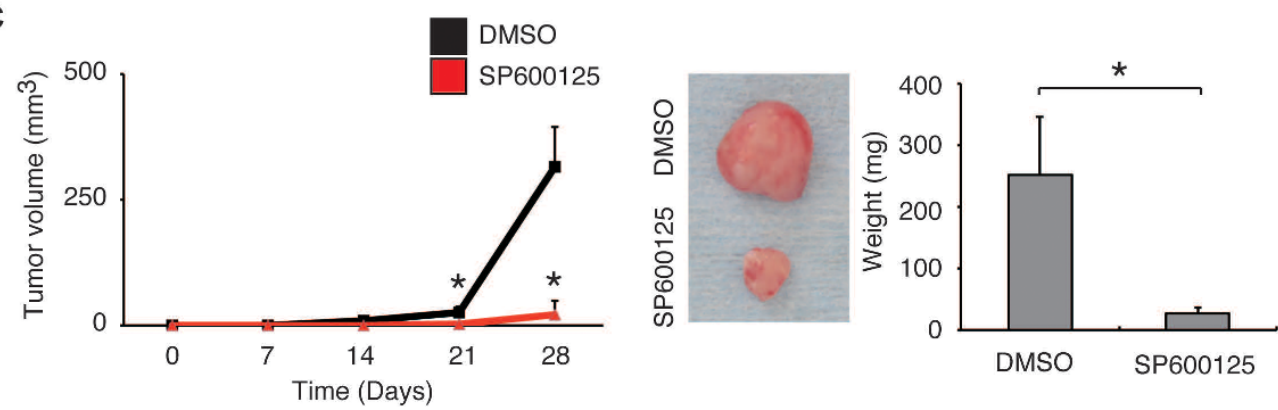

d
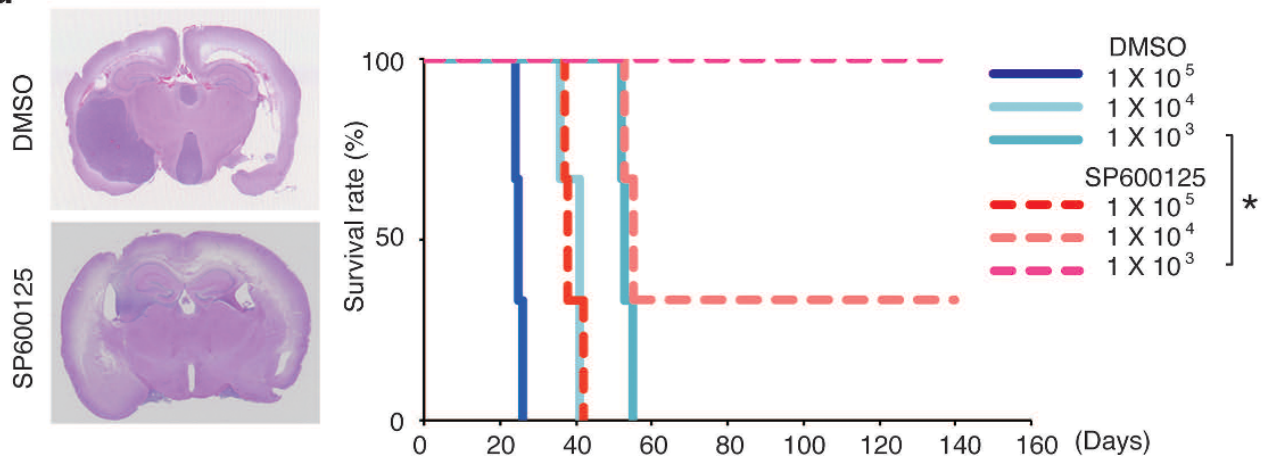

Figure 4 Transient in vivo JNK inhibition deprives stem-like glioblastoma cells of tumour-initiating potential and depletes established tumours of self-renewing and tumour-initiating glioblastoma cell populations. (a) Mice implanted subcutaneously with stem-like glioblastoma cells (TGS01, $1 \times 10^{6}$ cells) were administered intraperitoneal injection of the control vehicle (DMSO) or SP600125 (40 mg/kg/day), for 5 days starting on the next day of implantation ( 3 mice per group). Left, tumour volume at the indicated time points (mean \pm s.d.). Right, representative images of mice 28 days after implantation. ${ }^{*} P<0.05$. (b-d) Mice subcutaneously implanted with stem-like glioblastoma cells (TGS01, $1 \times 10^{6}$ cells) were administered, after tumour formation (tumour volume range, 224-364 mm $\mathrm{mm}^{3}$ ), intraperitoneal injection of the control vehicle (DMSO) or SP600125 (40 mg/kg/day) for 5 days (3 mice per group). On the next day of the final drug treatment, mice were sacrificed and dissociated tumour cells were subjected to serial sphere formation assays (b). Left, photomicrographs of the spheres (scale bars, $200 \mu \mathrm{m}$ ). Right, number of spheres formed (mean \pm s.d. of triplicate cultures derived from a single tumour of each treatment group). Essentially identical results were obtained from analysis of the remaining 2 tumours of each group. Alternatively, the dissected tumour cells $\left(1 \times 10^{6}\right.$ cells per mouse) were transplanted subcutaneously into the right flank ( 5 mice per group) (c). Left, tumour volume at the indicated time points (mean \pm s.d.). Right, at the end of the observation period ( 28 days after transplantation), mice were sacrificed and the weight of the secondary tumours was measured. Middle, representative image of the secondary tumours derived from the SP600125- and control-treated primary tumours. Serial dilutions of the dissected tumour cells derived from the SP600125- and control-treated primary tumours were also transplanted intracranially (d). Right, survival as evaluated by Kaplan-Meier analysis ( 3 mice per group). Left, representative images of haematoxylin and eosin staining of the brain sections of mice that had undergone transplantation of cells $\left(1 \times 10^{4}\right)$ from primary tumours treated with SP600125 or the control vehicle (DMSO) and were sacrificed at 20 days after transplantation. $* P<0.05$. 
a

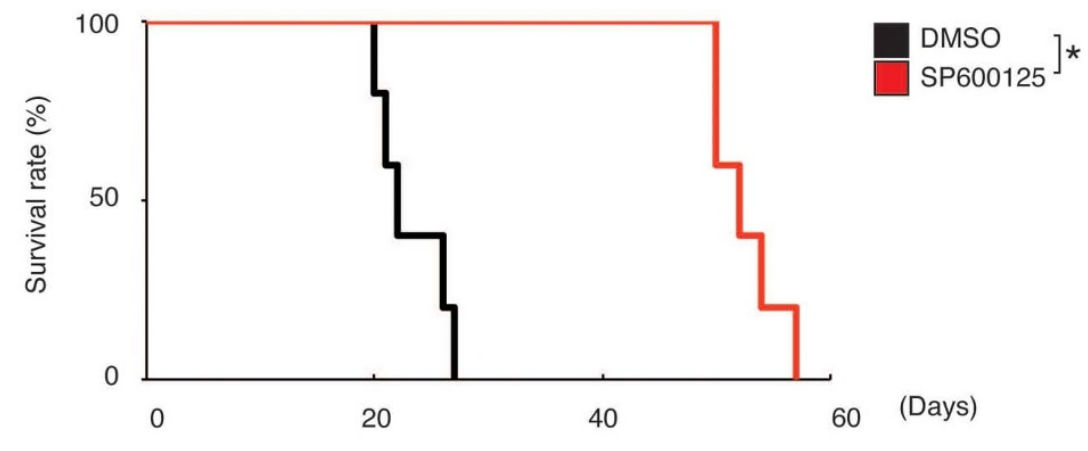

b

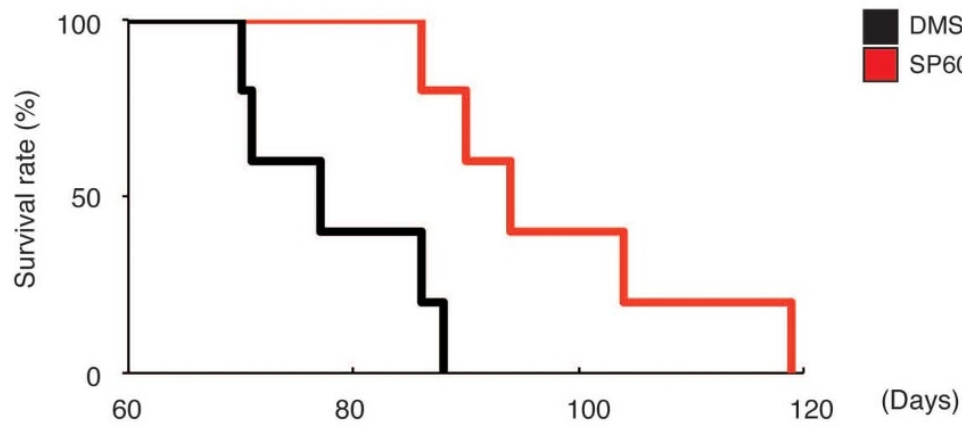

C
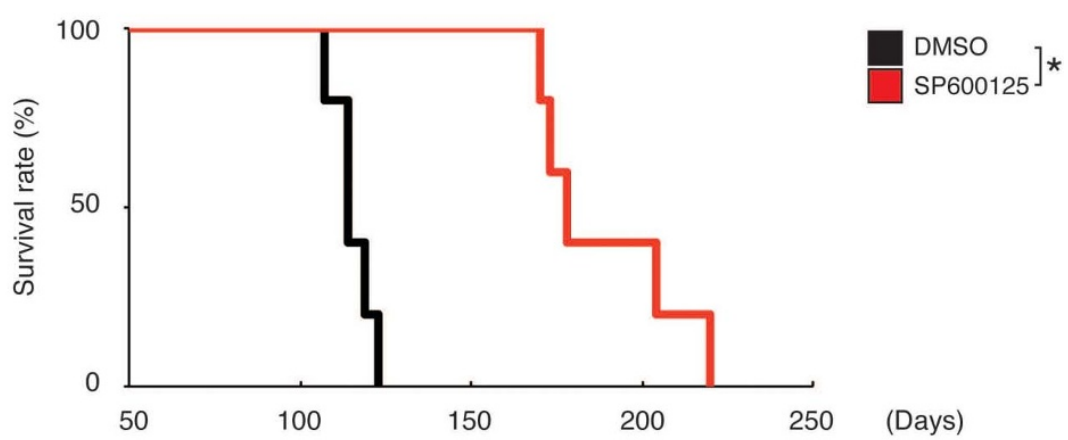

d

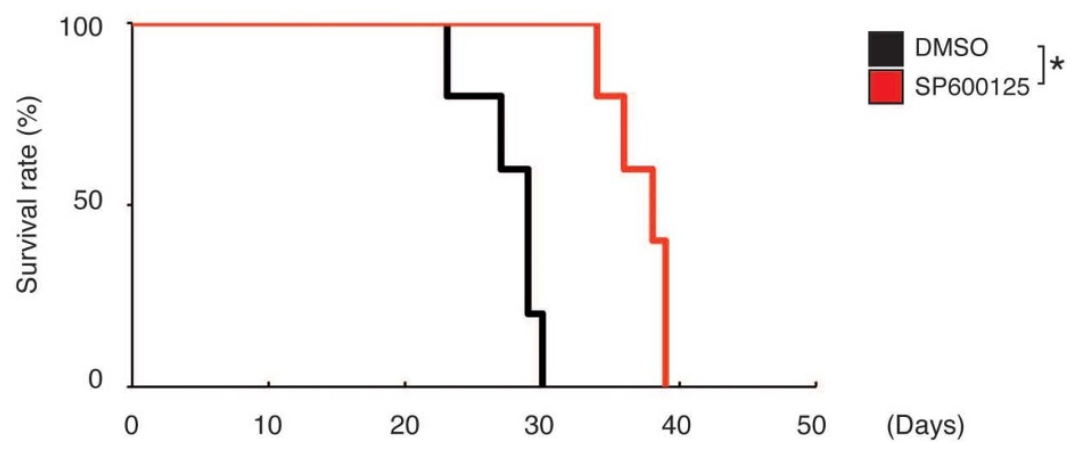

Figure 5 Prevention of brain tumour formation by stem-like glioblastoma cells via transient, systemic administration of JNK inhibitor. KaplanMeier plots showing survival of mice ( 5 mice per group) implanted intracranially with $1 \times 10^{4}$ stem-like glioblastoma cells ((a) TGS01, (b) GS-Y01, (c) TGS04, and (d) U87GS) followed by transient, systemic administration (intraperitoneal delivery, once daily for 10 consecutive days) of SP600125 (40 mg/ $\mathrm{kg} /$ day) or the control vehicle (DMSO). Drug administration began on the next day of intracranial implantation. ${ }^{*} P<0.05$.

potential while causing no or minimal adverse events or sequelae. To date, a number of molecules and/or pathways have been reported as potential targets in the control of tumour-initiating glioblastoma cells ${ }^{2,5}$. However, none has yet been proven to be a viable target of drugs meeting the above requirements. Here we have identified JNK as a critical regulator of the self-renewal and tumour-initiating potential of stem-like glioblastoma cells. Most importantly, our findings demonstrate that SP600125, an ATP-competitive, reversible inhibitor of JNK, is a potential candidate as a curative chemotherapeutic agent against glioblastoma. Indeed, systemic administration of SP600125, using a dosing schedule that retains ample room for intensification and improvement, was found to exert a significant anti-tumour effect against stem-like glioblastoma cells implanted into the brain without causing discernible adverse events. Our findings also suggest that the in vivo anti-tumour effect of SP600125 treatment most likely be attributed to the specific activity of SP600125 to deplete stem-like tumour cells and not to its non-specific growth inhibitory effect on bulk tumour cells. In support of this idea, the results of the serial transplantation assays demonstrated that short-term administration of a reversible inhibitor of JNK (i.e., SP600125) is sufficient to provide a long-lasting, preventive effect against secondary tumour formation (Fig. 4c, d). Furthermore, the 
results indicated that the in vivo SP600125 treatment depletes selfrenewing, stem-like cells but has virtually no impact on the bulk tumour cells (Fig. 4b and Supplementary Fig. 12). However, it needs to be acknowledged that these findings do not exclude the possibility that the tumour-initiating cells within established xenografts may not necessarily be stem-like cells and that SP600125 also targets such non-stem glioblastoma cells with tumour-initiating potential. Intriguingly, SP600125 is now increasingly delivered to the brain parenchyma via the intraventricular route in animal models of neurological diseases to improve biochemical and neurological functions, including cognitive function ${ }^{24-26}$. The reported neuroprotective activity of SP600125 makes it an even more attractive therapeutic option, and the reported findings also suggest that, in clinical settings, the drug could be administered not only systemically but also intrathecally, such as via an Ommaya reservoir installed during surgery.

It has been well documented that the JNK pathway is activated in astrocytic tumours in direct association with the WHO grade of malignancy but not in normal brain tissues, suggesting a role for JNK in the biology of astrocytic tumours including their most malignant form, glioblastoma ${ }^{27-29}$. While a previous study using the serum-cultured U87 cell line showed that JNK is indeed involved in the growth of bulk-cultured glioblastoma cells as well as xenografts derived from them, the results also showed that such JNK involvement is modest ${ }^{30}$. As this finding, which was also confirmed in this study (Supplementary Figs. 10 and 12), indicates that JNK inhibition would have only a modest effect on the growth of bulk glioblastoma cells, it alone may not support the use of JNK as a therapeutic target against glioblastoma. The identification of JNK as a key player in stem-like glioblastoma cells in this study, however, strongly supports use of JNK as a target of glioblastoma therapy. Of note, the JNK pathway could be activated by upstream events such as EGFR activation and PTEN loss, both of which occur frequently in glioblastoma $^{11,29,31,32}$. However, JNK2 $\alpha 2$, a JNK isoform constitutively activated through an autophosphorylation mechanism independently of upstream activating signals, is reportedly expressed in the majority of human glioblastomas ${ }^{30}$. Thus, targeting of the JNK pathway at or downstream of JNK may be warranted to control the pathway in glioblastoma cells.

We identified JNK in this study first as having a critical role in the regulation of the stem-like phenotypes of glioblastoma cells and subsequently demonstrated, as we initially assumed, its essential role in the maintenance of their tumour-initiating potential. Significantly, despite the fact that JNK inhibition was consistently "shortterm" in nature in this study (short-term treatment with a reversible inhibitor or transient siRNA transfection), some mice implanted with glioblastoma cells, which invariably leads to tumour development if untreated, survived without any evidence of tumour formation throughout extended observation periods when the implanted cells had undergone JNK inhibitor treatment. This observation indicates that the short-term JNK inhibition provided by the treatment was sufficient to lead tumour-initiating cells stably to transition into cells without tumour-initiating potential, and thus indicates that the deprivation of the tumour-initiating potential is a stable and robust state in the in vivo microenvironment whereas maintenance of tumour-initiating potential is an active state that requires continuous signalling. Whether the observed depletion of the tumour-initiating population is simply a long-lasting but essentially reversible event or a truly irreversible event may be a question difficult to address using animals that survive for 1-2 years at most. Nevertheless, long-term follow-up of the surviving mice in this study suggests that the possibility of tumour cells recovering their tumour-initiating potential is likely very low or nil. Thus, although the results of this study may not provide indisputable evidence of the hierarchy between tumour cells with and without tumour-initiating potential proposed by the cancer stem cell hypothesis, they clearly indicate that a molecule involved in the regulation of stem-like phenotypes is an attractive therapeutic target in gaining long-lasting control over the tumour-initiating population using short-term interventions.

In conclusion, we identified an essential role for JNK, a molecule aberrantly activated in glioblastoma, in the maintenance of the selfrenewal and tumour-initiating potential of stem-like glioblastoma cells. Short-term JNK inhibition both in vitro and in vivo resulted in selective, long-term depletion of tumour-initiating glioblastoma cells. In particular, systemic administration of the JNK inhibitor SP600125 successfully controlled tumour formation by stem-like glioblastoma cells implanted in the brain parenchyma without causing adverse events. Our results thus suggest JNK inhibition in combination with conventional, bulk tumour-directed therapies is a rational and promising strategy in the treatment of glioblastoma ${ }^{33}$. Our results also support the idea that targeting the regulatory mechanism of stem-like tumour cells is a viable approach toward realization of long-term control over cancer, irrespective of whether the cancer stem cell hypothesis is proven or remains a hypothesis. Intriguingly, the critical role of JNK in the maintenance of stem-like glioblastoma cells was reported by an independent group while this manuscript was in preparation ${ }^{34}$. Although this report by itself does not provide evidence that JNK is a superior therapeutic target compared to the candidate molecules previously proposed, the in vitro findings described in the report are consistent with and in support of those of this study, providing further support that JNK is a key regulator of stem-like glioblastoma cells. As such, the report reinforces our conclusion that JNK is an attractive target for therapeutic depletion of stem-like glioblastoma cells.

\section{Methods}

Reagents and antibodies. SP600125 was purchased from Calbiochem and used as dimethylsulfoxide (DMSO) solution. EGF and FGF2 were from PeproTech. AntiSox2 (MAB 2018), anti-glial fibrillary acidic protein (GFAP) (AF2594), and anti- $\beta$ IIItubulin (MAB 1199) were from R\&D. Anti-phospho-Akt (Ser473) (\#4098), anti-Akt (\#9272), anti-phospho-SAPK/JNK (Thr183/Tyr185) (\#9251), anti-phospho-c-Jun (Ser63) (\#9261), anti-c-Jun (\#9162), anti-phospho-p38 MAPK (Thr180/Try182) (\#4631), anti-p38 MAPK (\#9212), anti-phospho-ERK1/2 (\#9106), anti-ERK1/2 (\#4695), anti-PTEN (\#9552), anti-EGFR (\#4267), anti-FOXO1 (\#2880), anti-FOXO3 (2497), anti-FOXO4 (\#9427), and anti-PARP (\#2435) were from Cell Signaling Technology. Anti-nestin (AB5922) was from Chemicon. Anti-Bmil (05-637) was from Upstate. Anti-Musashi (ab212628) was from Abcam. Anti- $\beta$-actin (A5441) was from Sigma. Anti- $\alpha$-tubulin (CP06) and anti-p53 (OP43) were from Oncogene. AntiJNK1 (sc-474) and anti-JNK2 (sc-7345) were from Santa Cruz Biotechnology. Horseradish peroxidase (HRP)-conjugated secondary antibodies for immunoblotting were from Jackson ImmunoResearch and Upstate. Alexa Fluor 488and 594-conjugated secondary antibodies for immunocytochemistry were from Molecular Probes.

Serum-cultured glioblastoma cell lines. U87 and T98G cell lines were purchased from American Type Culture Collection (ATCC) and Riken Bioresource Center (Tsukuba, Japan), respectively. The U343 cell line was kindly provided by Dr. Mark L Rosenblum (University of California at San Francisco, USA). These cell lines were maintained in standard Dulbecco's Modified Eagle's Medium (DMEM) supplemented with $10 \%$ fetal bovine serum (FBS) and antibiotics (100 units/ml penicillin, $100 \mu \mathrm{g} / \mathrm{ml}$ streptomycin).

Isolation, culture, and characterization of stem-like glioblastoma cells. Isolation, establishment of patient-derived stem-like glioblastoma cells (GS-Y04, GS-Y05, GSNCC02, GS-NCC03) were carried out essentially as previously described in accordance with a protocol approved by the Institutional Review Boards of Yamagata University School of Medicine and the National Cancer Center, and the stem-like cells were maintained under the monolayer stem cell culture condition ${ }^{35-37}$. In brief, tumour tissues were washed in chilled sterile Hanks' balanced salt solution (HBSS) with $0.6 \%$ glucose and penicillin/streptomycin (PS), minced with scissors, and incubated in Accutase (Sigma) for $30 \mathrm{~min}$ at $37^{\circ} \mathrm{C}$. After being washed with HBSS/PS, the tissues were suspended in DMEM/F12 and filtered through a $70-\mu \mathrm{m}$ strainer (Falcon). The dissociated cells were cultured on non-coated dishes in the stem cell culture medium (DMEM/F12 serum-free medium [GIBCO] supplemented with B27 [Invitrogen], $20 \mathrm{ng} / \mathrm{ml} \mathrm{EGF,} 20 \mathrm{ng} / \mathrm{ml} \mathrm{FGF} 2,100$ units/ml penicillin, and $100 \mu \mathrm{g} / \mathrm{ml}$ streptomycin for all cell lines except for TGS01 and TGS04; DMEM/F12 serum-free medium supplemented with B27, $20 \mathrm{ng} / \mathrm{ml} \mathrm{EGF,} 20 \mathrm{ng} / \mathrm{ml} \mathrm{FGF2,} 35 \mathrm{mM}$ D-(+)glucose, $15 \mu \mathrm{g} / \mathrm{ml}$ insulin, and $2 \mathrm{mM} \mathrm{L}$-glutamine for TGS01 and TGS04, essentially according to the protocol of the original establisher of the cell lines ${ }^{38}$ ), and EGF and FGF2 were added to the culture medium every day. Under this culture condition, non-adherent cell aggregates referred to as tumourspheres were formed, which were 
mechanically dissociated and re-seeded at 3- to 4-day intervals. After 4 or 5 rounds of this passaging procedure, tumourspheres were plated onto collagen I-coated dishes (IWAKI) and maintained thereafter on collagen I-coated dishes with the stem cell culture medium (i.e., under the monolayer stem cell culture condition). In general, monolayer-cultured stem-like glioblastoma cells were dissociated mechanically or using Accutase and re-seeded once every 6 to 7 days.

Stem-like sublines of U87 and U343 (U87GS and U343GS, respectively) were established by culturing U87 and U343 cells on non-coated dishes in the stem cell culture medium (i.e., under the sphere culture condition), which led to formation of tumourspheres. The tumourspheres formed by U87 and U343 were treated as described for the establishment of patient-derived stem-like glioblastoma cells, to establish U87GS and U343GS, which were then maintained under the monolayer stem cell culture condition.

Isolation, establishment, and characterization of stem-like properties of patientderived stem-like glioblastoma cell lines TGS01, TGS04, GS-Y01, GS-Y02, GS-Y03 (previously designated \#38), and GS-NCC01 (previously designated SJ28P3) were conducted as previously described ${ }^{37-40}$. Stem-like properties (self-renewal capacity and multipotency of differentiation) of the 6 other cell lines (GS-Y04, GS-Y05, GS NCC02, GS-NCC03, U87GS and U343GS) described above were examined and verified in this study (see Supplementary Fig. 1). Differentiation potential was examined by culturing stem-like cells under the differentiation-inducing culture condition (DMEM/F12 containing 10\% FBS). Tumour-initiating potential (i.e., generation of glial brain tumours upon intracranial implantation) has been demonstrated in all cell lines tested to date (TGS01, TGS04, GS-Y01, GS-Y02, GS-Y03, GSY04, GS-NCC01, and U87GS). Analysis of protein expression of genes (EGFR, PTEN, and p53) undergoing frequent genetic alteration in glioblastomas ${ }^{41}$ was conducted by immunoblot analysis for the stem-like glioblastoma cell lines used in this study (Supplementary Fig. 17). The results indicated that none of them expressed a detectable level of PTEN, consistent with a previous report that lack of PTEN expression is a common feature of expandable stem-like glioblastoma cells ${ }^{42}$. Throughout the study, the cell number was determined using a haemocytometer and viability was examined using the dye exclusion method ( $0.1 \%$ trypan blue).

Sphere formation assay. For (primary) sphere formation assay, cells were dissociated and suspended in the stem cell culture medium at a density of $5 \times 10^{3}$ cells $/ \mathrm{ml}$ before $200 \mu \mathrm{l}$ of the cell suspension was transferred to each well of a non-coated 96 -well plate. For secondary sphere formation, primary tumourspheres formed by cells seeded onto non-coated $35-\mathrm{mm}$ dishes at a density of $5 \times 10^{3}$ cells $/ \mathrm{ml}$ in the stem cell culture medium were collected 3 days after seeding and dissociated before $200 \mu$ lof the cell suspension was transferred to each well of a non-coated 96-well plate. Tertiary sphere formation assay was similarly performed using cell suspension from secondary tumourspheres formed on non-coated $35-\mathrm{mm}$ dishes by cells from the primary tumourspheres seeded at a density of $5 \times 10^{3}$ cells $/ \mathrm{ml}$. The number of tumourspheres formed on the 96-well plates was counted 3 days after seeding under a phase-contrast microscope.

Mouse xenograft models. To develop the subcutaneous xenograft model, cells suspended in $200 \mu \mathrm{l}$ of phosphate-buffered saline (PBS) were injected into the flank region of 5-week-old male BALB/cAJcl- $n u / n u$ mice (CLEA Japan, Inc). After implantation, the recipient mice were monitored for general health status and presence of subcutaneous tumours. Tumour volume was determined by measuring tumour diameters (measurement of 2 perpendicular axes of tumours) using a caliper

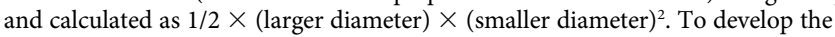
intracranial xenograft model, mice were anaesthetized with avertin $(0.3 \mathrm{~g} / \mathrm{kg}$ intraperitoneally) before cells suspended in $10 \mu \mathrm{l}$ of PBS were injected stereotactically into the right corpus striatum $(2.5 \mathrm{~mm}$ anterior and $2.5 \mathrm{~mm}$ lateral to the bregma, and $3.0 \mathrm{~mm}$ deep) of 5 -week-old male BALB/cAJcl- $n u / n u$ mice. After implantation, the recipient mice were monitored for general health status and appearance of neurological symptoms. Where indicated, mice were euthanized for histological analysis of brain or subcutaneous tumour, measurement of tumour weight, serial transplantation, and/or various cellular analyses represented by sphere formation assay. For serial transplantation and cellular analyses, excised tumours were washed in chilled sterile HBSS with $0.6 \%$ glucose and PS, minced with scissors, and incubated in Accutase for $30 \mathrm{~min}$ at $37^{\circ} \mathrm{C}$. After being washed with HBSS/PS, the tissues were suspended in PBS and filtered through a $70-\mu \mathrm{m}$ strainer. After determination of cell number and viability, the single-cell suspension of tumour cells was subjected to subcutaneous/intracranial injection and to cellular analyses. All animal experiments were performed under a protocol approved by the Animal Research Committee of Yamagata University.

Systemic drug administration to mice. Systemic administration of SP600125 and temozolomide was performed by intraperitoneal injection of drugs in $200 \mu \mathrm{l}$ DMSO solution. Control mice were administered the same volume $(200 \mu \mathrm{l})$ of drug-free DMSO.

Gene silencing by siRNA. siRNAs against human JNK1 (HSS108547), JNK2 (HSS108550), and FOXO1 (HSS103718), and Stealth RNAi ${ }^{\mathrm{TM}}$ siRNA Negative Control Duplexes (Medium GC Duplexes \#2) were purchased from Invitrogen. Transfection of siRNAs was performed using monolayer-cultured cells and Lipofectamine 2000 or Lipofectamine RNAi MAX (Invitrogen) according to the manufacturer's instruction.
Immunoblot analysis. Cells were lysed in the lysis buffer (10 mM Tris- $\mathrm{HCl}$ [pH 7.4], $0.1 \%$ SDS, $1 \%$ sodium deoxycholate, $0.15 \mathrm{M} \mathrm{NaCl}, 1 \mathrm{mM}$ EDTA, and $1 \%$ protease inhibitor cocktail set III [Calbiochem]). For analysis of phosphorylated proteins, cells were lysed in the lysis buffer supplemented with phosphatase inhibitors $(20 \mathrm{mM} \beta$ glycerophosphate, $10 \mathrm{mM} \mathrm{NaF}$, and $0.1 \mathrm{mM} \mathrm{Na}_{3} \mathrm{VO}_{4}$ ). After determination of protein concentration using the BCA Protein Assay Kit (Pierce), cell lysates containing equal amounts of protein were separated by SDS-PAGE and transferred to a polyvinylidene difluoride membrane. The membrane was probed with a primary antibody and then with an appropriate HRP-conjugated secondary antibody according to the protocol recommended by the manufacturer of each antibody. Blots were visualized using Immobilon Western Chemiluminescent HRP Substrate (Millipore).

Immunofluorescence. Cells plated onto coated glass coverslips were fixed with $4 \%$ paraformaldehyde in PBS for $15 \mathrm{~min}$ at room temperature. The fixed cover slips were permeabilized in $0.5 \%$ Triton X-100 for 5 min, washed twice in PBS, and incubated in a blocking solution (PBS containing $2 \%$ bovine serum albumin and $2 \%$ FBS) for $30 \mathrm{~min}$. The cells were then incubated in the blocking solution with primary antibodies at $4{ }^{\circ} \mathrm{C}$ overnight, followed by incubation with Alexa Fluor 488- or Alexa Fluor 594-conjugated secondary antibodies in the blocking solution for $1 \mathrm{~h}$ at room temperature. Stained cells were observed under an epifluorescence microscope (CKX41, Olympus) equipped with a CCD camera system (DP-30BW, Olympus). Fluorescence images were processed using Photoshop 6.0 (Adobe).

Y-maze test. The Y-maze test examines spontaneous alternation behaviour, which reflects spatial working memory but is currently regarded as a sensitive indicator of brain lesions, including hippocampal lesions, rather than a specific indicator of memory function alone ${ }^{43-45}$. Y-maze tests in this study were conducted in a maze consisting of $3 \mathrm{arms}$ ( $40 \mathrm{~cm}$ long, $9.5 \mathrm{~cm}$ high, and $4 \mathrm{~cm}$ wide, labelled A, B, or C, respectively) diverging at a $120^{\circ}$ angle from the central point essentially as described by Washida $\mathrm{K}$ et $\mathrm{al}^{46}$. A mouse was considered to have entered an arm when all 4 paws were positioned in the arm runway. An actual alternation was defined as entry into all 3 arms on consecutive occasions. The maximum alternation was subsequently calculated by measuring the total number of arm entries and subtracting 2 , and the percentage of alternation was calculated as (actual alternation/maximum alternation) $\times 100$. The total number of arms entered during the sessions, which reflected locomotor activity, was also recorded. For each set of Y-maze tests, mice underwent 1 session ( $=8$-min of Y-maze task) per day for consecutive 10 days, and average alternation behaviour (\%) and number of entries per session were determined.

Subcellular fractionation. Cells were homogenized in hypotonic buffer $(10 \mathrm{mM}$ Tris- $\mathrm{HCl}$ [pH 7.8], $150 \mathrm{mM} \mathrm{NaCl}$, and $1 \mathrm{mM}$ EDTA) containing $0.1 \%$ Triton X$100^{47}$. Lysates were centrifuged at $3,000 \mathrm{rpm}$ for $10 \mathrm{~min}$ at $4{ }^{\circ} \mathrm{C}$ and separated into pellet and supernatant fractions. The pellet fraction was re-suspended in hypotonic buffer containing $0.1 \%$ Triton X-100, re-centrifuged, and used as the nuclear fraction. The supernatant fraction was re-centrifuged at $15,000 \mathrm{rpm}$ for $20 \mathrm{~min}$ at $4{ }^{\circ} \mathrm{C}$ and used as the cytoplasmic fraction.

Statistical analysis. Results are expressed as mean \pm s.d., and differences were compared using the 2 -tailed Student's $t$-test. Mouse survival was evaluated using the Kaplan-Meier method and analysed using the log-rank test. Statistical analyses were performed using Microsoft Office Excel 2010 software (Microsoft Corp.). $P$ values less than 0.05 were considered statistically significant and indicated with asterisks $(*)$ in the figures.

1. Stupp, R. et al. Radiotherapy plus concomitant and adjuvant temozolomide for glioblastoma. N Engl J Med 352, 987-996 (2005).

2. Cheng, L., Bao, S. \& Rich, J. N. Potential therapeutic implications of cancer stem cells in glioblastoma. Biochem Pharmacol 80, 654-665 (2010).

3. Lathia, J. D., Venere, M., Rao, M. S. \& Rich, J. N. Seeing is believing: are cancer stem cells the Loch Ness monster of tumor biology? Stem Cell Rev 7, 227-237 (2011).

4. Neman, J. \& Jandial, R. Decreasing glioma recurrence through adjuvant cancer stem cell inhibition. Biologics 4, 157-162 (2010).

5. Tabatabai, G. \& Weller, M. Glioblastoma stem cells. Cell Tissue Res 343, 459-465 (2011).

6. Grossman, S. A. \& Batara, J. F. Current management of glioblastoma multiforme. Semin Oncol 31, 635-644 (2004).

7. Wen, P. Y. \& Kesari, S. Malignant gliomas in adults. N Engl J Med 359, 492-507 (2008).

8. Mangiola, A. et al. Glioblastoma therapy: going beyond Hercules Columns. Expert Rev Neurother 10, 507-514 (2010).

9. Papadopoulos, M. C., Saadoun, S., Davies, D. C. \& Bell, B. A. Emerging molecular mechanisms of brain tumour oedema. Br J Neurosurg 15, 101-108 (2001).

10. Sanai, N., Alvarez-Buylla, A. \& Berger, M. S. Neural stem cells and the origin of gliomas. N Engl J Med 353, 811-822 (2005)

11. Furnari, F. B. et al. Malignant astrocytic glioma: genetics, biology, and paths to treatment. Genes Dev 21, 2683-2710 (2007).

12. Muldoon, L. L. et al. Chemotherapy delivery issues in central nervous system malignancy: a reality check. J Clin Oncol 25, 2295-2305 (2007).

13. Dick, J. E. Stem cell concepts renew cancer research. Blood 112, 4793-4807 (2008). 
14. Clevers, H. The cancer stem cell: premises, promises and challenges. Nat Med 17, 313-319 (2011).

15. Stiles, C. D. \& Rowitch, D. H. Glioma stem cells: a midterm exam. Neuron $\mathbf{5 8}, 832-$ 846 (2008).

16. Gutkind, J. S. Regulation of mitogen-activated protein kinase signaling networks by G protein-coupled receptors. Sci STKE 2000, rel (2000).

17. Bennett, B. L. et al. SP600125, an anthrapyrazolone inhibitor of Jun N-terminal kinase. Proc Natl Acad Sci U S A 98, 13681-13686 (2001).

18. Zhang, D., Song, L., Li, J., Wu, K. \& Huang, C. Coordination of JNK1 and JNK2 is critical for GADD45alpha induction and its mediated cell apoptosis in arsenite responses. J Biol Chem 281, 34113-34123 (2006).

19. Sunayama, J. et al. FoxO3a functions as a key integrator of cellular signals that control glioblastoma stem-like cell differentiation and tumorigenicity. Stem Cells 29, 1327-1337 (2011).

20. Gross, N. D. et al. Inhibition of Jun NH2-terminal kinases suppresses the growth of experimental head and neck squamous cell carcinoma. Clin Cancer Res 13, 5910-5917 (2007).

21. Beier, D. et al. Temozolomide preferentially depletes cancer stem cells in glioblastoma. Cancer Res 68, 5706-5715 (2008).

22. Wang, W. et al. SP600125, a new JNK inhibitor, protects dopaminergic neurons in the MPTP model of Parkinson's disease. Neurosci Res 48, 195-202 (2004).

23. Morgan, L. et al. Development of a pentylenetetrazole-induced seizure model to evaluate kinase inhibitor efficacy in the central nervous system. Neurosci Lett 395 , 143-148 (2006).

24. Wang, Y. et al. JNK inhibitor protects dopaminergic neurons by reducing COX-2 expression in the MPTP mouse model of subacute Parkinson's disease. J Neurol Sci 285, 172-177 (2009).

25. Sharma, N., Deshmukh, R. \& Bedi, K. L. SP600125, a competitive inhibitor of JNK attenuates streptozotocin induced neurocognitive deficit and oxidative stress in rats. Pharmacol Biochem Behav 96, 386-394 (2010).

26. Ramin, M., Azizi, P., Motamedi, F., Haghparast, A. \& Khodagholi, F. Inhibition of JNK phosphorylation reverses memory deficit induced by beta-amyloid (1-42) associated with decrease of apoptotic factors. Behav Brain Res 217, 424-431 (2011)

27. Antonyak, M. A. et al. Elevated JNK activation contributes to the pathogenesis of human brain tumors. Oncogene 21, 5038-5046 (2002).

28. Assimakopoulou, M., Kondyli, M., Gatzounis, G., Maraziotis, T. \& Varakis, J. Neurotrophin receptors expression and JNK pathway activation in human astrocytomas. BMC Cancer 7, 202 (2007).

29. Li, J. Y. et al. Constitutive activation of c-Jun $\mathrm{N}$-terminal kinase correlates with histologic grade and EGFR expression in diffuse gliomas. J Neurooncol 88, 11-17 (2008).

30. Cui, J. et al. c-Jun NH(2)-terminal kinase 2alpha2 promotes the tumorigenicity of human glioblastoma cells. Cancer Res 66, 10024-10031 (2006).

31. Vivanco, I. et al. Identification of the JNK signaling pathway as a functional target of the tumor suppressor PTEN. Cancer Cell 11, 555-569 (2007).

32. Rong, Y. et al. Epidermal growth factor receptor and PTEN modulate tissue factor expression in glioblastoma through JunD/activator protein-1 transcriptional activity. Cancer Res 69, 2540-2549 (2009).

33. Rasheed, Z. A., Kowalski, J., Smith, B. D. \& Matsui, W. Concise review: Emerging concepts in clinical targeting of cancer stem cells. Stem Cells 29, 883-887 (2011).

34. Yoon, C. H. et al. c-Jun N-terminal kinase has a pivotal role in the maintenance of self-renewal and tumorigenicity in glioma stem-like cells. Oncogene, doi:10.1038/ onc.2011.634 (2012)

35. Pollard, S. M. et al. Glioma stem cell lines expanded in adherent culture have tumor-specific phenotypes and are suitable for chemical and genetic screens. Cell Stem Cell 4, 568-580 (2009).

36. Fael Al-Mayhani, T. M. et al. An efficient method for derivation and propagation of glioblastoma cell lines that conserves the molecular profile of their original tumours. J Neurosci Methods 176, 192-199 (2009).
37. Sunayama, J. et al. Crosstalk between the PI3K/mTOR and MEK/ERK pathways involved in the maintenance of self-renewal and tumorigenicity of glioblastoma stem-like cells. Stem Cells 28, 1930-1939 (2010).

38. Ikushima, H. et al. Autocrine TGF-beta signaling maintains tumorigenicity of glioma-initiating cells through Sry-related HMG-box factors. Cell Stem Cell 5, 504-514 (2009).

39. Sunayama, J. et al. Dual blocking of mTor and PI3K elicits a prodifferentiation effect on glioblastoma stem-like cells. Neuro Oncol 12, 1205-1219 (2010).

40. Sato, A. et al. MEK-ERK Signaling Dictates DNA-Repair Gene MGMT Expression and Temozolomide Resistance of Stem-Like Glioblastoma Cells via the MDM2-p53 Axis. Stem Cells 29, 1942-1951 (2011).

41. Comprehensive genomic characterization defines human glioblastoma genes and core pathways. Nature 455, 1061-1068 (2008).

42. Chen, R. et al. A hierarchy of self-renewing tumor-initiating cell types in glioblastoma. Cancer Cell 17, 362-375 (2010).

43. Lalonde, R. The neurobiological basis of spontaneous alternation. Neurosci Biobehav Rev 26, 91-104 (2002).

44. Hughes, R. N. The value of spontaneous alternation behavior (SAB) as a test of retention in pharmacological investigations of memory. Neurosci Biobehav Rev 28, 497-505 (2004).

45. Reisel, D. et al. Spatial memory dissociations in mice lacking GluR1. Nat Neurosci 5, 868-873 (2002).

46. Washida, K. et al. Nonhypotensive dose of telmisartan attenuates cognitive impairment partially due to peroxisome proliferator-activated receptor-gamma activation in mice with chronic cerebral hypoperfusion. Stroke 41, 1798-1806 (2010).

47. Shinzawa, K. \& Tsujimoto, Y. PLA2 activity is required for nuclear shrinkage in caspase-independent cell death. J Cell Biol 163, 1219-1230 (2003).

\section{Acknowledgements}

We thank Drs. Tomoki Todo and Nobuhito Saito at the University of Tokyo for generously providing us with the TGS cell lines, and also thank Dr. Motoo Nagane at Kyorin University for his valuable advice on xenograft experiments. We thank the members of our lab for their fruitful discussion and technical advice, and Dr. Tomoko Kagawa for her continuous support and encouragement. This work was supported by Grants-in-Aid for Scientific Research, for Challenging Exploratory Research, and for Young Scientists from the Ministry of Education, Culture, Sports, Science and Technology of Japan; by a Grant-in-Aid from the Global COE Program of the Japan Society for the Promotion of Science; by the National Cancer Center Research and Development Fund (23-A-20) and a grant from the Japan Brain Foundation.

\section{Author contributions}

C.K. and A.S. conceived the project, supervised all research, and wrote the manuscript. C.K., A.S., and K.M. designed the experiments. C.K., A.S., K.M., and M.O. performed the data analysis and produced the text and figures. A.S., K.M., K.Shibuya, S.Seino, K.Suzuki, and E.W. performed the experiments. Y.N., S. Shibui, and T.K. provided patient materials and clinical input. K.M. and A.S. contributed equally to this work.

\section{Additional information}

Supplementary information accompanies this paper at http://www.nature.com/ scientificreports

Competing financial interests: The authors declare no competing financial interests.

License: This work is licensed under a Creative Commons

Attribution-NonCommercial-NoDerivative Works 3.0 Unported License. To view a copy of this license, visit http://creativecommons.org/licenses/by-nc-nd/3.0/

How to cite this article: Matsuda, K. et al. Targeting JNK for therapeutic depletion of stem-like glioblastoma cells. Sci. Rep. 2, 516; DOI:10.1038/srep00516 (2012). 